\title{
Fuerzas armadas y narcotráfico: una aventura peligrosa. El caso mexicano y sus implicaciones para Argentina*
}

\section{Sergio Gabriel Eissa**}

\begin{abstract}
RESUMEN
Este artículo propone dos rutas de análisis. En primer lugar, se analiza la situación del tráfico de drogas en Argentina y las alternativas legales para involucrar a las fuerzas armadas. En función de esto último, y siguiendo el argumento predominante de que un aumento de los costos institucionales para el mercado de drogas disminuiria la oferta, se infiere qué consecuencias produciría el involucramiento de tropas militares. En segundo lugar, se analiza lo sucedido en México con el gradual aumento de los costos institucionales, que culminaron con el involucramiento de las Fuerzas Armadas en la lucha directa contra el narcotráfico. Ambas rutas sólo nos muestran señales de alarmas que deberian tomarse en cuenta al implementar una política.
\end{abstract}

\section{PALABRAS CLAVE}

Narcotráfico, guerra contra las drogas, fuerzas armadas, Argentina, México.

\begin{abstract}
This article proposes two routes of analysis. First, the situation of drug trafficking in Argentina and the legal alternatives to involve the armed forces are analyzed. Based on this last point, and following the predominant argument that an increase in institutional costs for the drug market would decrease the supply, it is inferred what consequences would result from the involvement of military troops. Second, what happened in Mexico is analyzed with the gradual increase in institutional costs, which culminated in the involvement of the armed forces in the direct fight against drug trafficking. Both routes only show alarm signals that should be taken into account when implementing a policy.
\end{abstract}

\section{KEYWORDS}

Police Function, Citizen Security, Human Rights, Public Ethics.

\footnotetext{
*Artículo recibido el 30 de noviembre de 2017 y aceptado para su publicación el 24 de mayo de 2018

**Profesor en la Facultad de Ciencias Sociales de la Universidad de Buenos Aires, Argentina. (seissa@yahoo.com) orcid.org/0000-0001-7530-8753
} 


\section{SUMARIO}

1. Introducción

2. Situación del narcotráfico en Argentina

3. Las "opciones" de intervención de las Fuerzas Armadas en la lucha contra el narcotráfico en Argentina

4. Potenciales consecuencias de la intervención de las Fuerzas Armadas desde una mirada economicista

5. La perspectiva comparada: el caso mexicano

6. Reflexión final: más sombras que luces

\section{INTRODUCCIÓN}

La "guerra contra las drogas" ha fracasado. El presidente Juan Manuel Santos sostuvo en la sesión extraordinaria de la Asamblea General de Naciones Unidas (UnGASS, por sus siglas en inglés) que el mundo debe entender que los métodos represivos y de penalización están fracasando. Expuso que es hora de darles a los Estados mayor flexibilidad para actuar de acuerdo con sus realidades internas y la posibilidad de que desarrollen políticas más autónomas, que les permitan enfrentar un fenómeno que ha causado millones de muertos alrededor del mundo. Asimismo, esta política prohibicionista sólo se ha enfocado en los eslabones más bajos de la cadena del narcotráfico, en vez de concentrarse en atacar a las cabezas de las organizaciones criminales.

Pese a lo expuesto, supongamos por unos minutos que esta política ha sido exitosa en el mundo, que nuestro país debe seguir ese modelo e involucrar a las Fuerzas Armadas para hacer frente a este flagelo, cuya gravedad no podemos desconocer. ¿Cuáles podrían ser los resultados?

En primer lugar, este artículo analiza la situación del narcotráfico en Argentina, es decir las acciones que supusieron el involucramiento indirecto de las Fuerzas Armadas, y se analizan las alternativas legales para la utilización de los militares de manera directa, en función del plexo normativo vigente. A partir de ello, y siguiendo el argumento predominante de que mayores costos institucionales disminuirían la oferta de drogas, se utiliza el marco analítico de Gary Becker con el fin de inferir potenciales consecuencias para ese país. En segundo lugar, y ya desde una perspectiva comparada, se estudia el caso mexicano para visualizar cuáles han sido las consecuencias del involucramiento de las Fuerzas Armadas en la lucha contra el narcotráfico. Finalmente, se presentan las reflexiones finales. 


\section{Situación del narcotráfico en Argentina}

Uno de los principales problemas para abordar la problemática del narcotráfico en Argentina es la ausencia de un buen diagnóstico. ${ }^{1}$ Por ello, resulta necesario recurrir a indicadores indirectos, para inferir las características que presenta el problema en ese país.

Un primer indicador al que podría recurrirse es la cantidad de detenidos en relación con la Ley 23.737 de drogas. De acuerdo con un trabajo de investigación realizado por el Centro de Estudios Latinoamericanos sobre Inseguridad y Violencia de la Universidad Nacional de Tres de Febrero, ${ }^{2}$ la población carcelaria argentina se incrementó en 157\%, entre 1973 y 2012, mientras que en la provincia de Buenos Aires la cifra fue de 252\%. Cabe destacar que el sistema penitenciario bonaerense ${ }^{3}$ y el federal representan $60 \%$ del país.

Mientras en el año 2002 78.3\% de los detenidos no tenía condena, en el 2012, ese valor era de 56.1\%. A nivel federal y en el total país esas cifras se mantienen en torno a 55\%, en promedio. En cuanto al tipo de delito, 53.4\% estaba detenido por robo, $19.4 \%$ por homicidio y $10.1 \%$ por delitos contra la Ley 23.737 de drogas. Sin embargo, de ese total, 41.3\% estaba privado de libertad por venta y $46.2 \%$ por transporte. Asimismo, 78\% de los detenidos por delitos contra la ley de drogas fueron apresados en flagrancia.

Lo anterior nos permite concluir que la inteligencia y la investigación criminal es pobre, por lo cual el sistema actúa preferentemente sobre los eslabones más débiles de la cadena del narcotráfico. ${ }^{4}$ Según datos de la Procunar (Procuraduría de Narcocriminalidad), 33\% de los delitos cometidos (en prisión preventiva y condenados) fueron contra la ley de drogas en el año 2015.

Cuadro 1. Delitos contra la ley de drogas 23.737 y otros (2015)

\begin{tabular}{|l|c|}
\hline \multicolumn{1}{|c|}{ Bien jurídico } & $\%$ \\
\hline Contra la propiedad & $39 \%$ \\
\hline Ley 23.737 & $33 \%$ \\
\hline Contra las personas & $11 \%$ \\
\hline Contra la libertad & $5 \%$ \\
\hline
\end{tabular}

\begin{tabular}{|l|c|}
\hline \multicolumn{1}{|c|}{ Bien juridico } & cantidad \\
\hline Contra la propiedad & 4515 \\
\hline Ley 23.737 & 3746 \\
\hline Contra las personas & 1301 \\
\hline Contra la libertad & 601 \\
\hline
\end{tabular}

\footnotetext{
1 Tokatlan, Juan Gabriel, "Un plan antidrogas fuera de época", Clarín, 5 de septiembre, 2016. [Consulta: 14 de noviembre, 2017]. Disponible en: https://www.clarin.com/opinion/plan-antidrogas-epoca_0_BJ01Pgqj.html

${ }^{2}$ Bergman, Marcelo, Masello, Diego y Arias, Christian, Delito, marginalidad y desempeño institucional en la Argentina: resultados de la encuesta de presos condenados, Buenos Aires, Untref - CELIV, 2014.

${ }^{3}$ Provincia de Buenos Aires.

${ }^{4}$ Bergman, Marcelo, et al., op. cit.
} 


\begin{tabular}{|l|c|}
\hline Contra la integridad sexual & $5 \%$ \\
\hline $\begin{array}{l}\text { Contra la administración } \\
\text { pública }\end{array}$ & $2 \%$ \\
\hline Contra la seguridad pública & $2 \%$ \\
\hline Otras leyes & $1 \%$ \\
\hline (En blanco) & $1 \%$ \\
\hline Contra la fe pública & $1 \%$ \\
\hline Contra el orden público & $1 \%$ \\
\hline $\begin{array}{l}\text { Contra el orden económico y } \\
\text { financiero }\end{array}$ & $0 \%$ \\
\hline Contra el Estado civil & $0 \%$ \\
\hline $\begin{array}{l}\text { contra la seguridad de la } \\
\text { nación }\end{array}$ & $0 \%$ \\
\hline Total & $100 \%$ \\
\hline
\end{tabular}

\begin{tabular}{|l|c|}
\hline Contra la integridad sexual & 564 \\
\hline $\begin{array}{l}\text { Contra la administración } \\
\text { pública }\end{array}$ & 206 \\
\hline Contra la seguridad pública & 199 \\
\hline Otras leyes & 123 \\
\hline (en blanco) & 101 \\
\hline Contra la fe pública & 76 \\
\hline Contra el orden público & 60 \\
\hline $\begin{array}{l}\text { Contra el orden económico y } \\
\text { financiero }\end{array}$ & 5 \\
\hline Contra el Estado civil & 5 \\
\hline $\begin{array}{l}\text { Contra la seguridad de la } \\
\text { nación }\end{array}$ & 4 \\
\hline Total & 11506 \\
\hline
\end{tabular}

Fuente: Análisis de Información y Planificación Operativa de Procunar, con datos obtenidos de SNEEP 2015 (http://datos. jus.gob.ar/dataset/sneep).

Las incautaciones de drogas también son otro indicador indirecto del problema. No obstante, si se comparan las cifras de incautaciones con el resto de los países de Sudamérica, se observa un mismo patrón. Por tanto, se puede concluir que la oferta, especialmente de cannabis, se ha incrementado en los últimos años (véanse gráficos adjuntos). ${ }^{5}$

De acuerdo con Mariana Souto Zabaleta y Paula Delfino, más allá del señalamiento de las Naciones Unidas (onU) en torno al tráfico y al crecimiento de los mercados de consumo, esta organización también comenzó a hacer "referencia a la aparición del procesamiento ilícito de cocaína". ${ }^{6}$ Tales informes de la onU se corresponden con los datos referidos al aumento del consumo de alcohol y de estupefacientes. ${ }^{7}$ Asimismo, estos datos son compatibles con una mayor

\footnotetext{
5 Souto Zabaleta, Mariana y Delfino, Paula, "La idea de una Argentina sin narcotráfico en un mundo libre de drogas: desafíos para el diseño de políticas públicas", ponencia para el XIII Congreso Nacional de Ciencia Política "La política en entredicho. Volatilidad global, desigualdades persistentes y gobernabilidad democrática", Sociedad Argentina de Análisis Político - Universidad Torcuato Di Tella, 2-5 de agosto, 2017, pp. 6 a 9.

${ }^{6} / d e m$, p. 18.

${ }^{7}$ Idem, pp. 17-21; Gorgal, Diego, "El problema de las drogas y las respuestas de políticas públicas", ponencia presentada en la Mesa Redonda "Contextualización del trabajo operativo: las funciones de la Gendarmería Nacional y las capacidades requeridas para los oficiales", Buenos Aires, Escuela Superior del Instituto Universitario de la Gendarmería Nacional, 9 de noviembre, 2017.
} 
disponibilidad en los mercados locales ${ }^{8}$ de las sustancias provenientes de otros países de Sudamérica, pero también -en el caso de las drogas de diseño- de Europa. ${ }^{9}$

Gráfico. 1

Argentina: incautaciones de cocaína

(bases, sales y crack)

1980-2015

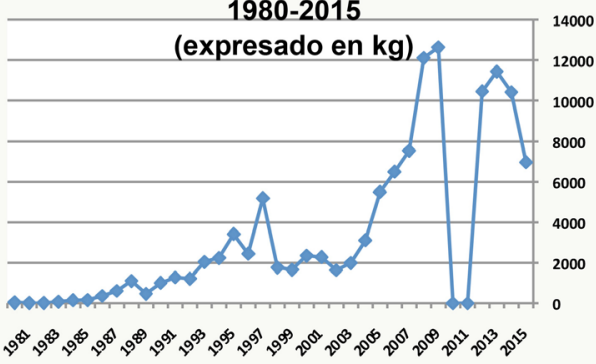

Fuente: Souto Zabaleta, Mariana y Delfino, Paula, “La idea de una Argentina sin narcotráfico en un mundo libre de drogas: desafíos para el diseño de políticas públicas”, ponencia para el XIII Congreso Nacional de Ciencia Política "La política en entredicho. Volatilidad global, desigualdades persistentes y gobernabilidad democrática”, Sociedad Argentina de Análisis Político - Universidad Torcuato Di Tella, 2-5 de agosto, 2017, p. 6.

Gráfico. 2

Incautaciones de marihuana

(1980-2015)

(expresado en kg)

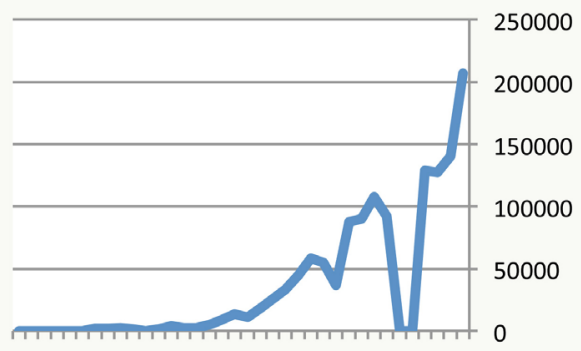

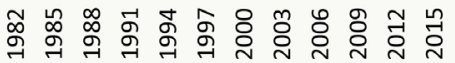

Fuente: Souto Zabaleta, Mariana y Delfino, Paula, "La idea de una Argentina sin narcotráfico en un mundo libre de drogas: desafios para el diseño de políticas públicas", ponencia para el XIII Congreso Nacional de Ciencia Política "La política en entredicho. Volatilidad global, desigualdades persistentes y gobernabilidad democrática”, Sociedad Argentina de Análisis Político - Universidad Torcuato Di Tella, 2-5 de agosto, 2017, p. 9.

${ }^{8}$ Souto Zabaleta, Mariana y Delfino, Paula, op. cit., pp. 21-23.

${ }^{9}$ Entrevista reservada con Fuente A, 6 de noviembre, 2017. 
Gráfico 3. Rutas del tráfico de drogas en Argentina

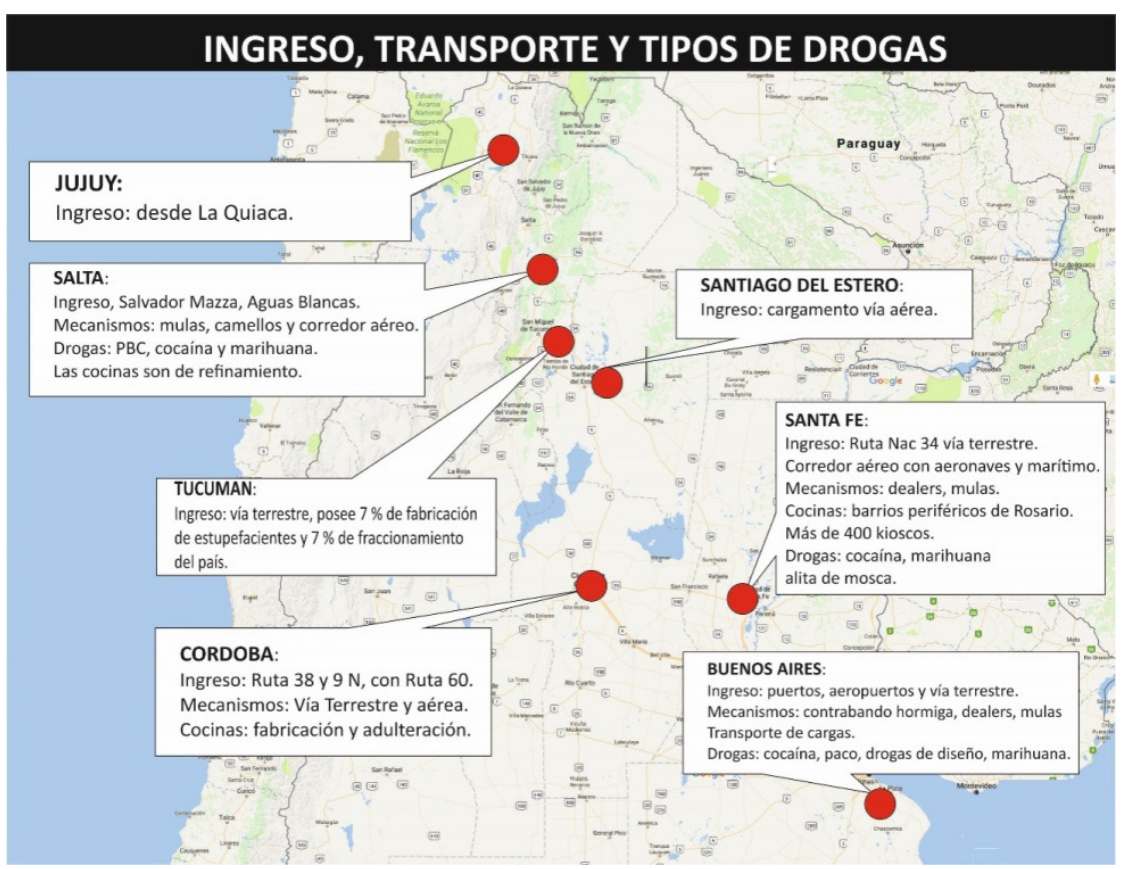

Fuente: Entrevista reservada con Fuente A, 6 de noviembre, 2017.

Finalmente, el territorio argentino resulta adecuado para el mercado de drogas, tanto por su infraestructura física y financiera, ${ }^{10}$ como por la tutela política y policial que permitió la expansión de las organizaciones criminales. ${ }^{11}$ Según Mariana Souto Zabaleta y Paula Delfino:

Es posible afirmar que el problema del narcotráfico en Argentina ha transitado en la última década una serie de transformaciones que se produjeron en dos etapas diferenciadas [...] En una primera etapa, al tradicional circuito de tránsito de clorhidrato de cocaína andina cuyo destino final es el mercado europeo, se agregó una nueva dinámica asociada al crecimiento de los mercados locales de consumo particularmente en los grandes centros urbanos del país. Una segunda etapa en

\footnotetext{
${ }^{10}$ Entrevista reservada Fuente B, 6 de noviembre, 2017.

"SAin, Marcelo, "El fracaso del control de las drogas ilegales en Argentina", Nueva Sociedad, 2009. [Consulta: 14 de noviembre, 2017]. Disponible enhttp://nuso.org/media/articles/downloads/3626_1.pdf
} 
la evolución del problema del narcotráfico aparece entonces una vez que se verifican las transformaciones descriptas en la primera etapa. El crecimiento del circuito de tránsito, así como del orientado al abastecimiento de los mercados locales, incorpora una mayor complejidad en la operatoria de las organizaciones criminales dedicadas a la comercialización ilícita de drogas. En el caso del macro-tráfico, aparece una más delicada división del trabajo con la formación de redes de colaboración segmentada, mayor capacidad de control del circuito de comercialización, de innovación y de cooptación de colaboradores locales. En el caso del micro-tráfico, la aparición de organizaciones locales más complejas, con dominio territorial y mayores índices de violencia asociados a su accionar. ${ }^{12}$

En este contexto, a través del Decreto 1407/2004 del Poder Ejecutivo Nacional, Argentina puso en marcha el Sistema Nacional de Vigilancia y Control Aeroespacial (Sinvica) con el objeto de controlar todos los movimientos en el espacio aéreo, a fin de cumplir las tareas de Defensa Aeroespacial y de Control del Tránsito Aéreo. Complementariamente, el Ministerio de Defensa, a través de la Resolución 206 del 16 de febrero de 2007, aprobó las "Acciones Contribuyentes al Fortalecimiento de las Capacidades de Vigilancia y Reconocimiento". En función del diseño y prioridades fijadas por las normas citadas, el Ministerio de Defensa desplegó algunos medios en el marco de la operación conjunta "Fortín”. Ello significó un incremento de la presencia de las Fuerzas Armadas con respecto al Operativo Pulqui, implementado en el año 2004..$^{13}$

Casi paralelamente, la Gendarmería Nacional ${ }^{14}$ fue desplegada en el Conurbano de la Provincia de Buenos Aires y en la Ciudad Autónoma de Buenos Aires a partir del año 2006 (Operativo Centinela), frente a la crisis de inseguridad que afectaba a dicha región. Se estima que en estos operativos han participado, en promedio, 12000 gendarmes por año. ${ }^{15}$

Cinco años más tarde, se puso en marcha el Operativo Escudo Norte, a través del decreto 1091/2011, en interacción con la Fuerza Aérea Argentina y el

\footnotetext{
12 Souto Zabaleta, Mariana y Delfino, Paula, op. cit., pp. 24.

${ }^{13}$ Entrevista reservada con Fuente $X, 18$ de noviembre, 2017.

${ }^{14}$ La Gendarmería Nacional, junto a la Prefectura Naval y la Policía de Seguridad Aeroportuaria son Fuerzas Federales de Seguridad o, en términos académicos, fuerzas intermedias. México no cuenta con este tipo de fuerzas, como la mayoría de los paises de América Latina. Véase BATAGLINo, JoRGE, "Fuerzas intermedias y lucha contra el tráfico de drogas: el caso de la Gendarmería en Argentina", uRvio - Revista Latinoamericana de Estudios de Seguridad, 2016. [Consulta: 14 de noviembre, 2017]. Disponible en http://revistas.flacsoandes.edu.ec/urvio/article/view/2228 ${ }^{15}$ Ibidem.
} 
Ejército Argentino, conforme la Resolución Conjunta del Ministerio de Defensa 821/2011 y del Ministerio de Seguridad, 905/2011. Este operativo fue prorrogado hasta principios del año 2016, cuando se aprobó el decreto 228/2016.

La aprobación de este último decreto incluyó un protocolo de derribo de tráfico aéreo irregular y permitió "reducir en 50\% los casos de tránsito aéreo irregular (TAI), como se denominan en la jerga aeronáutica los aviones interceptados sin planes de vuelo o en actitudes sospechosas". ${ }^{16} \mathrm{Al}$ respecto, el ministro de Defensa, Julio Martínez, agregó:

Las Fuerzas Armadas sólo aportan apoyo logístico. No van a intervenir en seguridad interior [...] Los militares colaboran de acuerdo a lo que permite la ley, en apoyo del control del aire con aviones y radares [...] El que involucraba a las Fuerzas Armadas en la lucha contra el narcotráfico era el kirchnerismo, que desplegaba al Ejército en el Norte, en el campo de acción, en contra de la ley. Nosotros los replegamos. ${ }^{17}$

En efecto, una vez que un vuelo ilegal es interceptado y se lo obliga a aterrizar, todo queda en manos de la Gendarmería, que debe movilizar sus efectivos por vía terrestre hacia el presunto lugar de aterrizaje de la aeronave o del lanzamiento de la droga. El procedimiento policial (law enforcement) es realizado por Fuerzas Federales de Seguridad.

Gráfico 4: Tráfico aéreo irregular en Argentina 2012-2017
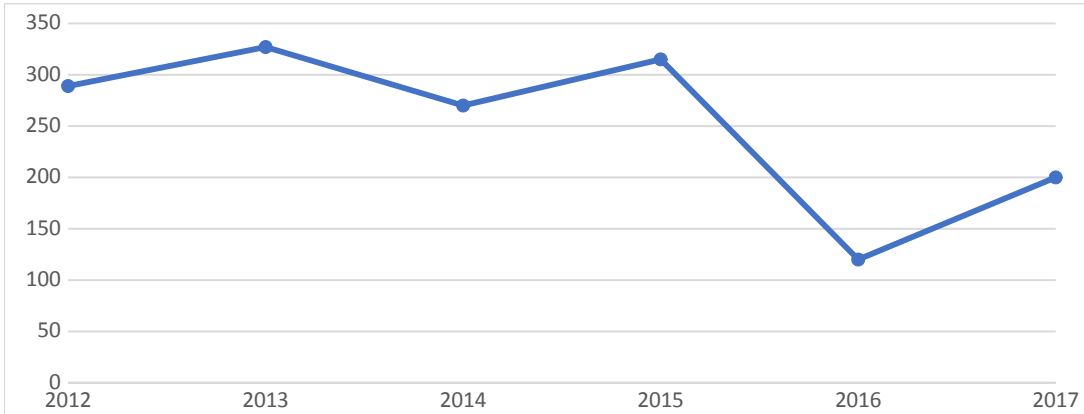

Fuente: Entrevista reservada con Fuente A, 6 de noviembre, 2017. Datos del año 2017 son parciales.

\footnotetext{
${ }^{16}$ De Vedia, Mariano, "Los militares apuestan al poder disuasivo en el control fronterizo", La Nación, 8 de agosto, 2016. [Consulta: 11 de noviembre, 2017]. Disponible en: http://www.lanacion.com.ar/1925858-los-militares-apuestan-al-poder-disuasivo-en-el-control-fronterizo

${ }^{17}$ Ibidem.
} 
La implementación del Operativo Fronteras implicó efectivamente el retiro de la frontera de unidades del Ejército que habían sido desplegadas durante la gestión de Cristina Kirchner (2011-2015). Pero la Fuerza Aérea Argentina modificó la definición de TAI. Hasta el año 2015, ésta incluía a todo el tráfico irregular: correspondía a contrabando y tráfico de drogas. En el año 2016, se establecieron dos categorías: los TAI sólo involucraban a los vuelos con droga y contrabando, mientras, los PIA (posible infractor aéreo) representaron 80\% (vuelos fumigadores, sin planes de vuelo, etcétera). Es decir, los TAI no disminuyeron, sólo se trató de un cambio estadístico. ${ }^{18}$

Por otra parte, se reacondicionaron helicópteros Bell-212 del Ejército Argentino para Gendarmería, con el fin de que ésta pueda, por vía aérea, concurrir al lugar de aterrizaje o "bombardeo" de droga de manera más eficaz. Sin embargo, esas aeronaves no se pueden utilizar porque no hay organización operativa, pese a la inversión que se realizó en ellas. ${ }^{19}$ Finalmente, si bien se pregona que las Fuerzas Armadas sólo colaborarán en la lucha contra el narcotráfico con el apoyo logístico y el control del espacio aéreo, el gobierno de Mauricio Macri canceló la fabricación de la modernización del avión de ataque Pucará $^{20}$ en la Fábrica Argentina de Aviones (Fadea) y, en su lugar, se compraron aviones Texan T-6 en Estados Unidos. Pese a la información que brindó el Ministerio de Defensa, éstos no sirven para derribar a los TAI, dado que sólo están preparados para que los pilotos de la Fuerza Aérea aprendan a volar. ${ }^{21}$

\section{Las “opciones” de intervención de las Fuerzas Armadas en la lucha CONTRA EL NARCOTRÁFICO EN ARGENTINA}

De acuerdo con la legislación vigente, la intervención de las Fuerzas Armadas en la lucha contra el narcotráfico en Argentina podría ser encuadrada en tres

\footnotetext{
${ }^{18}$ Entrevista reservada con Fuente B, 6 de noviembre, 2017.

${ }^{19}$ Entrevista reservada con Fuente $A, 6$ de noviembre, 2017.

${ }^{20}$ Entrevista reservada a Fuente D, 24 de febrero, 2017: "El T6C es el entrenador sin posibilidades de entrar en combate. Para reemplazar al Pucará debería ser el at que es idéntico al Super Tucano. Y puede portar equipos de vigilancia electrónica. El AT6 si es apto para la frontera norte H24. El T6 es una avioneta". Véase también IñuRRIETA, SeBASTIÁN, "Malestar en las Fuerzas Armadas por un duro plan de recortes de gastos", Cronista, 22 de marzo, 2017. [Consulta: 11 de noviembre, 2017]. Disponible en: https://www.cronista.com/economiapolitica/Malestar-en-las-Fuerzas-Armadas-por-un-duro-plan-de-recorte-de-gastos-20170322-0049.html

21 "Cómo son los aviones militares Texan II que el gobierno le compró a EE. UU.", La Nación, 2 de octubre, 2017. [Consulta: 11 de noviembre, 2017]. Disponible en: http://www.lanacion.com.ar/2068426-como-son-los-aviones-militares-texan-ii-que-el-gobierno-le-compro-a-eeuu; Entrevista reservada con Fuente C, 11 de noviembre de 2017. Además, la compra molestó a Brasil, según Agullera, Edgardo, "Brasil, molesto por la selección de avión de EE.UU. para entrenar a la Fuerza Aérea", Cronista, 13 de febrero, 2017. [Consulta: 11 de noviembre, 2017]. Disponible en: http:// www.ambito.com/872557-brasil-molesto-por-seleccion-de-avion-de-eeuu-para-entrenar-a-la-fuerza-aerea
} 
opciones. La primera (C1) supondría la realización de inteligencia estratégica criminal por parte de las Fuerzas Armadas, dentro y fuera del territorio, a los efectos de detectar y prevenir el accionar del delito transnacional dedicado al negocio del narcotráfico. Esto requiere una modificación de la Ley 25.520 y sus modificatorias, de Inteligencia Nacional.

La segunda opción (C2) está prevista en nuestra legislación y se ha venido aplicando en los últimos años. Ésta es la colaboración del Sistema de Defensa Nacional con el Sistema de Seguridad Interior. Así, cuando las Fuerzas Armadas -en tanto que ejercen el control del aeroespacio- detectan un tránsito aéreo irregular (TAI), que no constituye una amenaza estatal militar externa (AEME) o una amenaza contra los objetivos de valor estratégico, comunican a la Gendarmería Nacional la presencia de dicha aeronave, y se activa un protocolo para su intercepción. De esta manera, se busca que dicho vuelo abandone nuestro espacio aéreo o se sigue y ubica el lugar de aterrizaje y se informa a las Fuerzas Federales de Seguridad (Gendarmería, Prefectura y la Policía de Seguridad Aeroportuaria), responsables primarias de la lucha contra los delitos transnacionales, para que actúen.

La tercera opción (C3) requiere que el Congreso de la Nación apruebe una ley de derribo. ${ }^{22}$ Si eso sucediera, la diferencia con $\mathrm{C} 1$, sería que las Fuerzas Armadas, a través de la Fuerza Aérea Argentina, y siguiendo un estricto protocolo, podrían derribar ese TAI.

La cuarta opción (C4) requeriría la modificación de la Ley 23.554 de Defensa Nacional y la Ley 24.059 de Seguridad Interior, y supondría, como en el caso de México y Colombia, el involucramiento de tropas de combate en un enfrentamiento directo contra los grupos participantes en el negocio ilícito del narcotráfico.

Se debe aclarar que el adiestramiento, la adquisición de medios, la modificación de la doctrina y de inteligencia requieren, un cambio en las normas vigentes porque, caso contrario, supondría una violación de la Ley de Seguridad Interior.

\footnotetext{
${ }^{22}$ El Decreto 228 del 21 de enero de 2016 aprobó en su artículo 9 las "Reglas de Protección Aeroespacial". Sin embargo, es discutible la validez constitucional de este decreto, en tanto que no modifica ni puede modificar la Ley de Seguridad Interior 24.059 .
} 


\section{Potenciales consecuencias de la intervención de las Fuerzas Armadas DESDE UNA MIRADA ECONOMICISTA ${ }^{23}$}

En un trabajo del año 1974, Gary Becker ${ }^{24}$ sostuvo que si se mantienen constantes todos los factores explicativos del delito (psicológico, social, etcétera) y se incrementa la probabilidad de ser capturado, la cantidad de delitos disminuiría. Simplificando el argumento de Gary Becker, la ganancia que obtiene un delincuente por cometer cierto delito sería igual a la ganancia bruta, menos la probabilidad de ser capturado y condenado.

Esta probabilidad de que el delincuente sea penado es subjetiva, entonces, independiza fuertemente la percepción criminal de la gravedad de la pena. Si el castigo es muy alto - por ejemplo, la pena de muerte-, pero la percepción de ser atrapado y condenado es muy baja, el criminal percibirá que su "costo" de delinquir es bajo y la acción estatal tendrá poca capacidad disuasoria. En consecuencia, un delincuente decidirá ingresar al negocio de la droga si la ganancia neta es superior a los beneficios que obtendría realizando otra actividad, delictiva o no.

Así, en $\mathrm{Gn}=\mathrm{Gb}-\mathrm{Ci}$, el "premio" o "ganancia neta” (Gn) de la actividad delictiva es el resultado de la "ganancia bruta" del negocio ilícito, menos la acción punitiva estatal o "costos institucionales" (Ci). Este planteamiento implica el supuesto de que a Ci mayores se puede disminuir el "premio", o sea Gn, de la actividad delictiva: $\mathrm{Si} \mathrm{Ci} 2>\mathrm{Ci} 1=>\mathrm{Gn} 2<\mathrm{Gn} 1$.

Siguiendo la formulación de aquellos que proponen involucrar a las Fuerzas Armadas en la lucha contra el narcotráfico, si se aumentan los costos institucionales (Ci), a los que se enfrentaría un delincuente como consecuencia de aumentar el poder de fuego del Estado frente a este problema, involucrando no sólo a las Fuerzas de Seguridad (Gendarmería, Prefectura Naval y Policía de Seguridad Aeroportuaria), sino también a las Fuerzas Armadas, disminuirían las ganancias netas de los narcotraficantes. Ello desalentaría el involucramiento de los individuos que, racionalmente, buscan realizar otro tipo de actividad que les reporte mayores ingresos. Es decir, la oferta de drogas disminuiría y, en consecuencia, también el consumo.

Sin embargo, esta argumentación no tiene en cuenta algunos aspectos clave del negocio ilícito del narcotráfico. En primer lugar, éste tiene dos características principales: la búsqueda del beneficio económico y, en virtud de

\footnotetext{
${ }^{23}$ Agradezco la colaboración de Jorge Souto para la redacción de este apartado.

${ }^{24}$ Becker, Gary, op. cit.
} 
que se trata de un negocio ilícito, los conflictos se resuelven por mecanismos extrajudiciales, es decir, el uso de la violencia. El nivel de esta última es un buen predictor del estadio en que se encuentra el problema del narcotráfico en una ciudad, región o país, y si existe más de una organización disputándose dicho mercado.

En segundo lugar, si analizamos el fenómeno como un negocio, con cierta lógica de mercado, debemos considerar que la demanda de droga es inelástica en relación con el precio. Esto significa que la cantidad demandada se reduce proporcionalmente menos que el aumento del precio. En efecto, la adicción tiene una alta resiliencia frente al aumento de los precios de la droga y actúa más al aumentar el "premio" (Gb) que al disminuir la demanda.

En tercer lugar, el prohibicionismo, las sanciones y los castigos sobre la oferta aumentan los costos operativos. Entonces, cuanto mayor sea el costo, ceteris paribus, mayor será el precio que deberán pagar los consumidores, cuya dificultad para disminuir el consumo (inelasticidad) los fuerza a pagar esos precios mayores. Así, esto puede derivar en una escalada de la violencia, eventualmente.

Volviendo entonces a las cuatro posibilidades de intervención de las Fuerzas Armadas en la lucha contra el narcotráfico, se puede plantearse que C4 > C3 $>$ C2 $>$ C1. En consecuencia, en la medida que se aumentan las acciones punitivas - hasta alcanzar el mayor grado de prohibición, que supone la utilización de tropas de combate de las Fuerzas Armadas-, mayores serán los costos de los delincuentes y mayores serán los precios para los consumidores.

Pero, como la demanda es inelástica, ésta no disminuirá y los beneficios se mantendrán constantes o aumentarán, es decir, habrá un "premio" mayor. Dado que el "premio" será mayor, los delincuentes estarán dispuestos a correr más riesgos para obtener dichas ganancias. Paralelamente, cuanto más sea el beneficio esperado, los criminales estarán más predispuestos a usar la violencia para apropiarse del negocio.

¿Estas conclusiones, que se derivan de un análisis teórico, son observables en aquellos países que han militarizado la lucha contra el narcotráfico?

\section{La PERSPECTIVA comparada: el caso meXicano}

Es imposible entender la narcocriminalidad en México sin atender a dos factores clave: por un lado, la extensa frontera que lo vincula con los Estados Unidos y, por otro, el sistema de partido único, dominado por el Partido Revolucionario Institucional (PRI). Este último rigió sin fisuras entre 1929 y 1989 y permitió el 
fortalecimiento de un Estado altamente centralizado que "favoreció las bases para el desarrollo del negocio del tráfico de drogas desde dentro del gobierno, a partir de los altos niveles de corrupción en todos los poderes del Estado, con concentración en niveles subnacionales". ${ }^{25}$

La consolidaron a los criminales mexicanos en la producción, tráfico y distribución de heroína ${ }^{26}$ fue posible gracias a los cambios económico-sociales que se produjeron en Estados Unidos; la demanda de nuevas drogas por parte de los jóvenes -en el marco del movimiento hippie a fines de los años sesenta y principios de los setenta-, así como la guerra de Vietnam (1965-1975). Además, el desmantelamiento del aparato político que ocasionó el PRI fue un proceso en el cual los narcotraficantes lograron una mayor autonomía. Pero fueron el neoliberalismo y la cultura yuppie de los años noventa los que permitieron que las organizaciones criminales mexicanas acumularan "un poderío financiero sin precedentes, gracias al control casi total de la oferta de cocaína dirigida hacia el vecino país del Norte" ${ }^{27}$.

Entre mediados de los años ochenta y principios de los noventa, Estados Unidos implementó una dura política para restringir el ingreso de cocaína colombiana a través del estado de la Florida. Ello obligó a las organizaciones criminales colombianas a asociarse con sus pares mexicanos para aprovechar la ventaja que ofrecía la frontera México-Estados Unidos. Alentados por el lucrativo negocio de la cocaína, comenzaron a trabajar por su cuenta, adquiriendo mayor capacidad de corrupción y de generar violencia. ${ }^{28}$

En 1997, un funcionario de la DEA (Drug Enforcement Administration) declaró al diario Wall Street Journal que en México no había ni una sola institución, con responsabilidad sobre el tráfico de drogas, en la que tuvieran confianza. ${ }^{29}$ De esta manera, en el marco de la Directiva de Seguridad Nacional firmada por Reagan en 1986, que declaró a los narcóticos como una preocupación de la seguridad nacional, el Gobierno mexicano comenzó a involucrar gradualmente a los militares en la lucha contra el narcotráfico. ${ }^{30}$

El primer paso lo efectuó el presidente Miguel de la Madrid (1982-1988), quien también estableció que el tráfico de drogas era un problema de seguridad

\footnotetext{
${ }^{25}$ CEPEDA, Julo, "Narcotráfico, amenaza irresuelta", Revista Debates Latinoamericanos, año 13, núm. 26, p. 3.

${ }^{26}$ Ibidem; Mazltell, Antonio, op. cit.

${ }^{27}$ MaZITELL, Antonio, op. cit.

${ }^{28}$ Freeman, Laurie y SierRa, Jorge Luis, "México: la trampa de la militarización", en Coletta Youngers, Eileen Rosin, Drogas y democracia en América Latina. El impacto de la política de Estados Unidos, Buenos Aires, Wola - Editorial Biblos, 2005. ${ }^{29}$ Ibidem.

${ }^{30}$ EISSA, SERGIO, Lucha contra el narcotráfico y su impacto en América Latina, Madrid, Editorial Académica Española, 2012.
} 
nacional. Éste consideraba que las policías estaduales estaban fuertemente corrompidas por las organizaciones criminales y que las Fuerzas Armadas eran las únicas capacitadas para enfrentar a los narcotraficantes, y eran un socio menos corrupto.

Posteriormente, el presidente Salinas de Gortari (1988-1994) incluyó a los militares en el proceso de toma de decisión en esta temática y, por su parte, Ernesto Zedillo (1994-2000) creó el Consejo Nacional de Seguridad Pública, dando participación a las Secretarías de Marina y Defensa. ${ }^{31}$

A principios del siglo XXI se produjeron algunos cambios en este negocio. En primer lugar, la restricción de la demanda de cocaína en los Estados Unidos y la producción de marihuana en ese país obligó a las organizaciones criminales mexicanas a competir por nuevas rutas y mercados. Ello desató una escalada de violencia en la cual intervinieron "milicias armadas locales", formadas por pandillas locales, exsubordinados y exmilitares, como los "Zetas". ${ }^{32}$

En segundo lugar, la corrupción institucional y la estructura federal que hacía recaer la responsabilidad primaria de la lucha contra la inseguridad en las gobernaciones, atomizando la respuesta institucional y la recolección de información para efectuar un adecuado análisis criminal, impulsaron al Gobierno federal a buscar una respuesta en la militarización para la lucha contra el narcotráfico.

En tercer lugar, el aumento de la ayuda militar y policial de Estados Unidos a México ha contribuido también al aumento de la violencia. La Iniciativa Mérida, firmada en el año 2008, no es el primer antecedente de colaboración entre Estados Unidos y México en la lucha contra el narcotráfico. ${ }^{33}$ No obstante, este acuerdo promovido por el Gobierno de México, el cual se basa en el concepto de responsabilidad compartida entre ambos países, preveía la formación y equipamiento para las Fuerzas Armadas y las policías involucradas. La principal crítica que se le hizo al plan fue su énfasis en la "seguridad", y no en el fortalecimiento de las instituciones mexicanas para enfrentar la problemática

\footnotetext{
31 Freeman, Laurie y Sierra, Jorge Luis, op. cit.

32 Ibidem.

${ }^{33}$ Como reseña Barreda Vidal, se destacan los siguientes antecedentes: la Operación Cóndor (1975); el Tratado de Cooperación entre los Estados Unidos Mexicanos y los Estados Unidos de Norteamérica sobre Asistencia Legal Mutua (1987); el Acuerdo entre los Estados Unidos Mexicanos y los Estados Unidos de América sobre Cooperación para Combatir el Narcotráfico y la Farmacodependencia (1989); el Grupo de Respuesta de la Frontera (1990); el Grupo de Alto Nivel de contacto para el Control de las Drogas (1996); la estrategia entre Estados Unidos y México para el combate al narcotráfico (1997), y la Alianza para la Seguridad y la Prosperidad de América del Norte (ASPAN) en 2005. Barreda Vidal, Pilar Zoraida, "La cooperación bilateral México-Estados Unidos contra la delincuencia organizada trasnacional en el marco de la Iniciativa Mérida", Revista IUS (MÉxICO), vol. 8, núm. 34, p. 44.
} 
de la droga. Por tal motivo, durante la presidencia de Barack Obama (20092017) se aprobó un nuevo plan llamado "Más allá de Mérida" que mantenía los objetivos iniciales, pero ampliaba el enfoque con el que se quería abordar esta problemática. ${ }^{34}$

Durante diez años, Estados Unidos entregó equipamiento por un valor de 1.600 millones de dólares, y transfirió 2300 millones de dólares en tecnología y capacitación. ${ }^{35}$ Sin embargo, la Iniciativa Mérida, incluida dentro del International Narcotics Control and Law Enforcement, no fue la única fuente de financiamiento a México. Por ello, todos los programas de financiamiento para la lucha contra el narcotráfico totalizaban para el año 2017 la suma de 2888 millones de dólares.

En cuarto lugar, existe un creciente tráfico ilegal de armas, desde los Estados Unidos a México, que terminan en manos de los narcotraficantes. De acuerdo con Alejandro Hope, "90\% de las armas del narco vienen de Estados Unidos", aunque otras estimaciones sitúan esa cifra en torno a 70\%. ${ }^{36}$

Por su parte, la violencia, medida a través de la tasa de homicidio, se incrementó significativamente a partir del involucramiento directo de las Fuerzas Armadas en la lucha contra las organizaciones criminales vinculadas al narcotráfico, pasando a ser una de las más altas de América Latina ${ }^{37}$ y el mundo. ${ }^{38} \mathrm{La}$ tasa de homicidio se elevó hasta alcanzar casi los 25 por cada 100000 habitantes en el año 2011, tal como se predecía en el marco analítico.

Pese al cambio en la estrategia, el aumento de la tasa de homicidio a partir del año 2015 y la prospectiva de que el año 2017 sería el peor en esta materia desde el 2011, refuerzan una vez más la convicción de que la llamada "guerra contra las drogas" ha fracasado.

Ahora bien, ¿por qué se observa una disminución de la tasa de homicidios entre 2012 y 2014? Una posible explicación es que los homicidios se esconden en las estadísticas de la cantidad de desaparecidos. Esto supondría que el

\footnotetext{
${ }^{34}$ Idem, p. 52.

35 "Diez años de la Iniciativa Mérida: un recuento de la cooperación entre México y los Estados Unidos", Expansión Nacional, 10 de abril, 2017. [Consulta: 5 de junio, 2017]. Disponible en: http://expansion.mx/nacional/2017/04/09/10anos-de-la-iniciativa-merida-un-recuento-de-la-cooperacion-entre-mexico-y-eu

${ }_{36}$ Pauller, Juan, "¿De dónde salen las armas pesadas del narco en México?, BBc Mundo, 6 de mayo, 2015. [Consulta: 5 de junio, 2017]. Disponible en: http://www.bbc.com/mundo/noticias/2015/05/150505_mexico_narcotrafico_carteles_origen_armas_jp

${ }^{37}$ Oficina de las Naciones Unidas contra la Droga y el Delto, "Estudio mundial sobre el homicidio", unodc, 2013. [Consulta: 7 de noviembre, 2017]. Disponible en https://www.unodc.org/documents/gsh/pdfs/GLOBAL_HOMICIDE_Report_ExSum_spanish.pdf

38 "Mexico's spiralling murder rate", IISS Analysis, 9 de mayo, 2017. [Consulta: 29 de mayo, 2017]. Disponible en: http://www.iiss.org/en/iiss\%20voices/blogsections/iiss-voices-2017-adeb/may-8636/mexico-murder-rate-9f41
} 
accionar del Estado ha profundizado su accionar ilegal, lo cual se refleja en parte en las ejecuciones, pero, sobre todo, en las desapariciones.

A tal punto es grave la cifra de desaparecidos durante el sexenio de Peña Nieto que el gobierno ha propuesto una modificación en la legislación para definir qué se entiende por "desaparecido". En efecto, según wolA ${ }^{39}$ "las desapariciones en México han incrementado a niveles alarmantes desde el 2007 (y muestran) una preocupante resistencia del gobierno a acabar con la opacidad que rodea la desaparición de más de 30000 personas en el país en los últimos años”.

Gráfico 5. Tasa de homicidios cada cien mil habitantes (enero-febrero, 1997-2017)

\section{ITASA DE ASESINATOS}

El índice registrado en enero y febrero de 2017 se ubicó en 3.06 homicidios por cada 100 mil habitantes; es el más alto para un inicio de año desde 2011, cuando fue de 3.07 delitos, según cifras del Sistema Nacional de Seguridad

Pública.

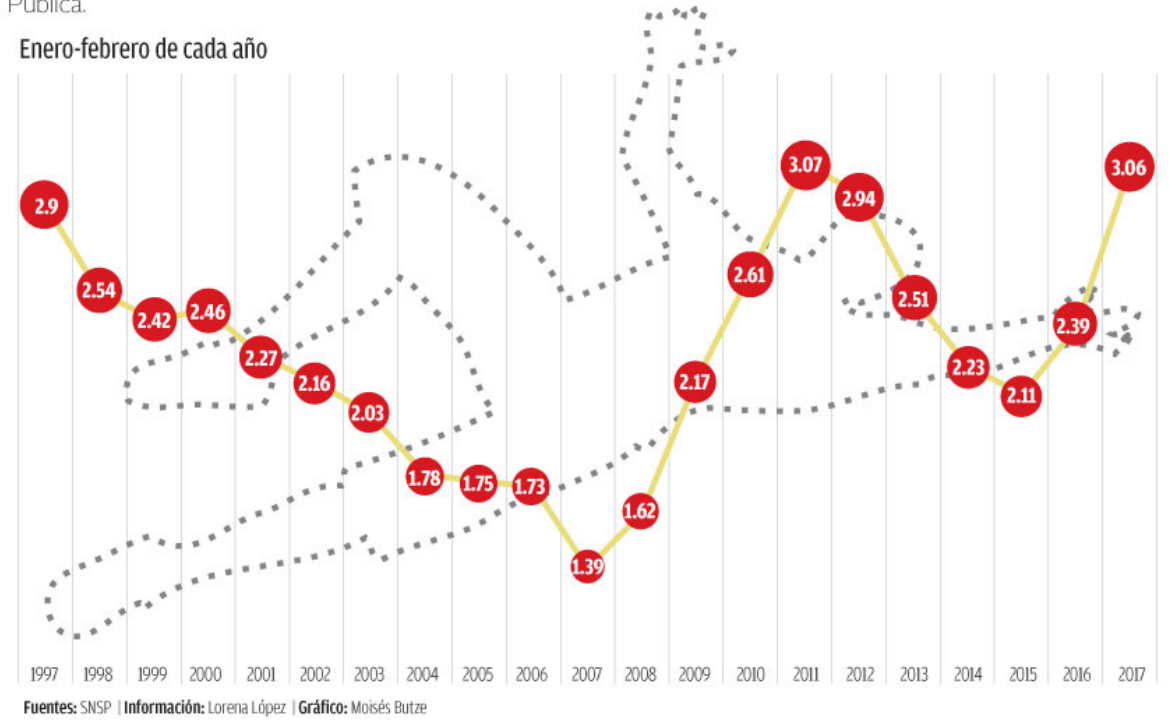

Fuente: LóPEz, LoRenA, "Homicidios: en 2017, el peor bimestre desde 2011”, Grupo Milenio, 22 de marzo, 2017. [Consulta: 28 de mayo, 2017]. Disponible en: http://www.milenio.com/policia/homicidios-2017-peor_primer_bimestre-2011-asesinatos-snsp-crimen_organizado-milenio_0_924507557.html

\footnotetext{
39 Washington Office on Latin America, "Cambios que propone el presidente Peña a la Ley de Desaparición de México serian un retroceso", wolA, 25 de abril, 2017. [Consulta: 29 de mayo, 2017]. Disponible en: https:/www.wola.org/ es/2017/04/cambios-que-propone-el-presidente-a-la-ley-de-desaparicion-de-mexico-serian-un-retroceso-wola/
} 
Gráfico 6. Tasa de homicidios y desapariciones cada cien mil habitantes

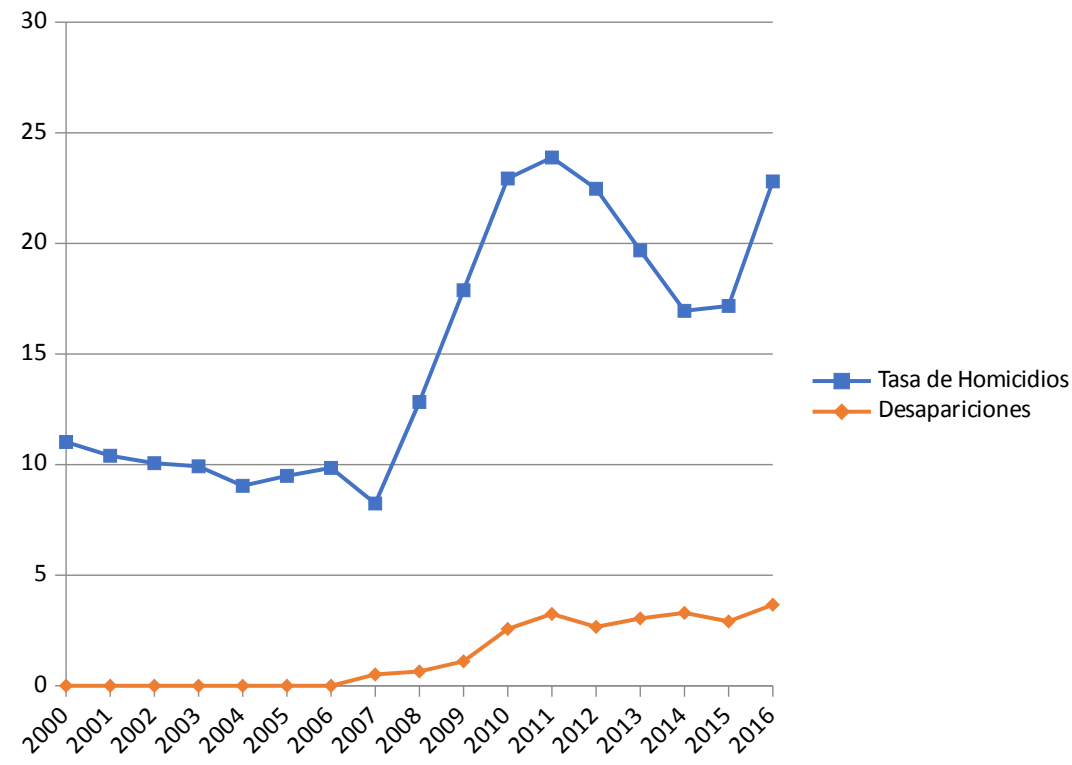

Fuente: elaboración propia con base en datos de IISS, Inegi, Sinais, PAHo y Secretariado Ejecutivo del Sistema Nacional de Seguridad Pública del Gobierno de México.

En resumen, y de acuerdo con lo hasta aquí expuesto, esta tragedia humanitaria no ha resultado en una disminución del tráfico ni del consumo. Encima, ha incrementado el nivel de violencia. Asimismo, estas acciones afectaron seriamente el control civil de las Fuerzas Armadas, la calidad democrática y los derechos humanos.

\section{REFLEXIÓN FINAL: MÁS SOMBRAS QUE LUCES}

La "guerra contra las drogas" ha fracasado y lo ha hecho desde el mismo momento en que Estados Unidos impulsó la militarización para la resolución de este grave problema, en 1986. De acuerdo con los informes de la Oficina de Naciones Unidas contra la Droga y el Delito, ${ }^{40}$ el consumo y el tráfico se han incrementado globalmente.

\footnotetext{
${ }^{40}$ UNODC, "Informe mundial sobre drogas", unODC, 2016. [Consulta: 8 de noviembre, 2017]. Disponible en: http://www. unodc.org/doc/wdr2016/V1604260_Spanish.pdf
} 
En cuanto a América Latina, y en función de los datos que compiló de distintas fuentes Marcelo Bergman, ${ }^{41}$ se observa que, en los países seleccionados, el consumo también ha aumentado. Tal como sostiene Juan Gabriel Tokatlian, ${ }^{42}$ América Latina no puede asumir como propia "la lógica de la guerra contra las drogas”, sin que el resto de los Estados alcancen un acuerdo social y político que aborde, al menos parcialmente, los problemas estructurales que han permitido que el narcotráfico florezca.

Pese a todo lo expuesto, durante los días de la campaña electoral presidencial del 2015, el deja vú de las nuevas amenazas ha vuelto a instalarse en el debate de la agenda pública argentina. Muchos candidatos, más o menos abiertamente, plantearon el involucramiento de las Fuerzas Armadas en la lucha contra el narcotráfico o, dicho de otra manera, la militarización de la respuesta frente al flagelo de la droga.

Esta situación no es nueva. Claramente, la experiencia comparada nos muestra que la utilización de las Fuerzas Armadas en la lucha contra el narcotráfico ha fracasado y ha provocado un aumento de la violencia en otros países, donde, también, se han corrompido las instituciones estatales y el instrumento militar de la defensa nacional.

Se ha argumentado que Argentina se está mejicanizando. Esto no es así en la actualidad, pero si se llegara a optar por involucrar a las Fuerzas Armadas de manera directa en la lucha contra el narcotráfico, sí será ése nuestro destino. ¿El camino de México es el que quiere recorrer nuestro país? Ciertamente no.

¿Existen alternativas? Trabajar fuertemente sobre el consumo; tener información certera sobre cuáles son las características de los consumidores (cómo se segmentan por tipo de droga, grupo etáreo, género, etcétera); tener estadísticas sobre cómo es el tráfico de drogas, las modalidades delictivas y las particularidades de las organizaciones en nuestro país, ${ }^{43}$ y mejorar la eficacia del sistema de seguridad y justicia.

Si la mayoría de los detenidos por violación a la ley de drogas con sentencia firme en nuestro país son consumidores, vendedores minoristas y transportistas, y en la mayoría de los casos son atrapados en flagrancia, jamás lograremos

\footnotetext{
${ }^{41}$ Bergman, Marcelo, Drogas, narcotráfico y poder en América Latina, Buenos Aires, Fondo de Cultura Económica, 2016, pp. 90-91.

${ }^{42}$ Tokatllan, JuAn Gabriel, Qué hacer con las drogas. Una mirada progresista sobre un tema habitualmente abordado desde el oportunismo político y los intereses creados, Buenos Aires, Siglo XXI, 2017, p. 10.

${ }^{43}$ Souto Zabaleta, Mariana, "El desarrollo de investigaciones empiricas en el estudio del problema del narcotráfico", ponencia presentada en las Primeras Jornadas de Estudios Sociales sobre Delito, Violencia y Policía: Ia seguridad en cuestión, La Plata, Universidad Nacional de La Plata, 2017.
} 
erradicar este flagelo. Como sostiene Marcelo Bergman, ${ }^{44}$ los eslabones más débiles de la cadena son fácilmente reemplazables por un numeroso "ejército de reserva”.

En otras palabras, necesitamos una eficaz inteligencia criminal, una agencia federal de investigaciones, una aduana incorrupta, una UIF que investigue el lavado de dinero, una AFIP que persiga a los grandes evasores y una justicia transparente y rápida que apunte a cortar la cabeza de Medusa. Esto requiere de una fuerte voluntad política de todos los actores políticos porque dicha cabeza no se encuentra en las villas, sino en el corazón financiero de nuestro país. Como dijo Arturo Illia: "A mí me derrocaron las veinte manzanas que rodean la Casa de Gobierno".

\section{Bibliografía}

Aguilera, Edgardo, "Brasil, molesto por la selección de avión de EE.UU. para entrenar a la Fuerza Aérea”, Cronista, 13 de febrero, 2017. [Consulta: 11 de noviembre, 2017]. Disponible en: http://www.ambito.com/872557-brasil-molesto-por-seleccion-de-avion-de-eeuu-para-entrenar-a-la-fuerza-aerea

BarReda Vidal, Pilar Zoraida, "La cooperación bilateral México-Estados Unidos contra la delincuencia organizada trasnacional en el marco de la Iniciativa Mérida”, RevisTa IUS (MÉxico), vol. 8, núm. 34.

Battaglino, Jorge, "Fuerzas intermedias y lucha contra el tráfico de drogas: el caso de la Gendarmería en Argentina”, uRvio - Revista Latinoamericana de Estudios de Seguridad, 2016. [Consulta: 14 de noviembre, 2017]. Disponible en http:// revistas.flacsoandes.edu.ec/urvio/article/view/2228

Bergman, Marcelo, Drogas, narcotráfico y poder en América Latina, Buenos Aires, Fondo de Cultura Económica, 2016.

Bergman, Marcelo, Masello, Diego y Arias, Christian, Delito, marginalidad y desempeño institucional en la Argentina: resultados de la encuesta de presos condenados, Buenos Aires, Untref - celiv, 2014.

CePedA, Julio, "Narcotráfico, amenaza irresuelta”, Revista Debates Latinoamericanos, año 13 , núm. 26.

“Cómo son los aviones militares Texan II que el gobierno le compró a EE. UU.", $L a$ Nación, 2 de octubre, 2017. [Consulta: 11 de noviembre, 2017]. Disponible en: http://www.lanacion.com.ar/2068426-como-son-los-aviones-militares-texanii-que-el-gobierno-le-compro-a-eeuu

De Vedia, Mariano, "Los militares apuestan al poder disuasivo en el control fronterizo", La Nación, 8 de agosto, 2016. [Consulta: 11 de noviembre, 2017]. Disponible en:

${ }^{44}$ Bergman, Marcelo, op. cit. 
http://www.lanacion.com.ar/1925858-los-militares-apuestan-al-poder-disuasivo-en-el-control-fronterizo

"Diez años de la Iniciativa Mérida: un recuento de la cooperación entre México y los

Estados Unidos”, Expansión Nacional, 10 de abril, 2017. [Consulta: 5 de junio, 2017]. Disponible en: http://expansion.mx/nacional/2017/04/09/10-anos-dela-iniciativa-merida-un-recuento-de-la-cooperacion-entre-mexico-y-eu

EISSA, Sergio, Lucha contra el narcotráfico y su impacto en América Latina, Madrid, Editorial Académica Española, 2012.

Freeman, Laurie y Sierra, Jorge Luis, “México: la trampa de la militarización”, en Coletta Youngers, Eileen Rosin, Drogas y democracia en América Latina. El impacto de la politica de Estados Unidos, Buenos Aires, Wola -Editorial Biblos, 2005.

Gorgal, DiEgo, "El problema de las drogas y las respuestas de políticas públicas”, ponencia presentada en la mesa redonda "Contextualización del trabajo operativo: las funciones de la Gendarmería Nacional y las capacidades requeridas para los oficiales”, Buenos Aires, Escuela Superior del Instituto Universitario de la Gendarmería Nacional, 9 de noviembre, 2017.

IÑURRIETA, SEBASTIÁN, "Malestar en las Fuerzas Armadas por un duro plan de recortes de gastos”, Cronista, 22 de marzo, 2017. [Consulta: 11 de noviembre, 2017]. Disponible en: https://www.cronista.com/economiapolitica/Malestar-en-las-Fuerzas-Armadas-por-un-duro-plan-de-recorte-de-gastos-20170322-0049.html

López, LorenA, "Homicidios: en 2017, el peor bimestre desde 2011", Grupo Milenio, 22 de marzo, 2017. [Consulta: 28 de mayo, 2017]. Disponible en: http://www. milenio.com/policia/homicidios-2017-peor_primer_bimestre-2011-asesinatos-snsp-crimen_organizado-milenio_0_924507557.html

“Mexico’s spiralling murder rate”, IIss Analysis, 9 de mayo, 2017. [Consulta: 29 de mayo, 2017]. Disponible en: http://www.iiss.org/en/iiss\%20voices/blogsections/ iiss-voices-2017-adeb/may-8636/mexico-murder-rate-9f41

Oficina de las Naciones Unidas contra la Droga y el Delito, "Estudio mundial sobre el homicidio”, unODC, 2013. [Consulta: 7 de noviembre, 2017]. Disponible en https://www.unodc.org/documents/gsh/pdfs/GLOBAL_HOMICIDE_Report_ExSum_spanish.pdf

PAullier, JuAn, “¿De dónde salen las armas pesadas del narco en México?, ввс Mundo, 6 de mayo, 2015. [Consulta: 5 de junio, 2017]. Disponible en: http://www. bbc.com/mundo/noticias/2015/05/150505_mexico_narcotrafico_carteles_origen_armas_jp

Saín, MARCELo, "El fracaso del control de las drogas ilegales en Argentina”, Nueva Sociedad, 2009. [Consulta: 14 de noviembre, 2017]. Disponible enhttp://nuso.org/ media/articles/downloads/3626_1.pdf

Souto Zabaleta, Mariana, "El desarrollo de investigaciones empíricas en el estudio del problema del narcotráfico", ponencia presentada en las Primeras Jornadas de Estudios Sociales sobre Delito, Violencia y Policia: la seguridad en cuestión, La Plata, Universidad Nacional de La Plata, 2017. 
Souto Zabaleta, Mariana y Delfino, Paula, “La idea de una Argentina sin narcotráfico en un mundo libre de drogas: desafíos para el diseño de políticas públicas”, ponencia para el XIII Congreso Nacional de Ciencia Política "La política en entredicho. Volatilidad global, desigualdades persistentes y gobernabilidad democrática”, Sociedad Argentina de Análisis Político - Universidad Torcuato Di Tella, 2-5 de agosto, 2017.

TOKatlian, JuAn Gabriel, Qué hacer con las drogas. Una mirada progresista sobre un tema habitualmente abordado desde el oportunismo politico y los intereses creados, Buenos Aires, Siglo XXI, 2017.

Tokatlian, Juan Gabriel, "Un plan antidrogas fuera de época”, Clarín, 5 de septiembre, 2016. [Consulta: 14 de noviembre, 2017]. Disponible en: https://www.clarin. com/opinion/plan-antidrogas-epoca_0_BJQ1Pgqj.html

UNODC, "Informe mundial sobre drogas", unODC, 2016. [Consulta: 8 de noviembre, 2017]. Disponible en: http://www.unodc.org/doc/wdr2016/V1604260_Spanish.pdf

Washington Office on Latin America, "Cambios que propone el presidente Peña a la Ley de Desaparición de México serían un retroceso”, wolA, 25 de abril, 2017. [Consulta: 29 de mayo, 2017]. Disponible en: https://www.wola.org/es/2017/04/ cambios-que-propone-el-presidente-a-la-ley-de-desaparicion-de-mexico-serian-un-retroceso-wola/ 
